\title{
THE WORD AND CONJUGACY PROBLEMS FOR THE KNOT GROUP OF ANY TAME, PRIME, ALTERNATING KNOT
}

\author{
C. M. WEINBAUM
}

\begin{abstract}
The decision problems in the title are solved using the solutions of the word and conjugacy problems obtained by Lyndon and Schupp, respectively, for certain classes of groups.
\end{abstract}

1. Introduction. Each tame knot $K$ has a knot group $G$ whose word problem is solvable by Waldhausen's result [6]. Here we give additional results when $K$ is also prime, alternating, and nontrivial. We show that the free product of $G$ and a free group on one generator $x_{0}$ is a group $H$ which falls in to one of the categories for which Lyndon [4] and Schupp [5] solved the word and conjugacy problems, respectively. ( $H$ has a presentation satisfying the properties $\mathrm{C}(4)$ and $\mathrm{T}_{3}[4$, p. 219].) We thereby obtain another solution of the word problem for $G$ and a solution of the conjugacy problem for $G$.

In the proof, the given knot is replaced with an equivalent one having a knot-diagram with special properties (referred to as a common knot-diagram). Presentations of $G$ and of another group $H$ are obtained from a knot-diagram, using Dehn's method [3, p. 157]. When the knot-diagram is common, the presentation of $H$ (as a factor group of a free group $F$ ) satisfies $\mathrm{C}(4)$ and $\mathrm{T}_{3}$. An automorphism of $F$ yields a second presentation of $H$ in which one generator $x_{0}$ is not mentioned in the defining relations. Adding the relation $x_{0}=1$ to this second presentation gives a presentation of $G$. Thus $H$ is a free product as claimed.

2. Common knot-diagrams. Let $K$ be a nontrivial polygonal knot in 3-space $R^{3}$. Let $K$ be the range of a continuous function $f$ defined on $[0,1]$, with $f(0)=f(1)$. Assume $K$ is in regular position with respect to the projection $p$ which sends $(x, y, z)$ to $(x, y, 0)$. (Knottheoretic terminology is taken from Crowell and Fox [2].) The knot-

Presented to the Society, October 10, 1970 under the title The word problem and tame, prime, alternating knots; received by the editors November 30, 1970.

AMS 1970 subject classifications. Primary 20F10, 20F05; Secondary 20E40, 55A05, $55 \mathrm{~A} 25$.

Key words and phrases. Generators and relations, word problem, conjugacy problem, fundamental group, knot group, knot-diagram, tame knot, prime knot, alternating knot. 
diagram $p(K)$ has $m \geqq 3$ double points $V_{1}, \cdots, V_{m}$ which are the projections of $2 m$ points of $K: f\left(t_{i}\right), 1 \leqq i \leqq 2 m$, for some $t_{i}$ in $[0,1]$. Assume $0<t_{1}<\cdots<t_{2 m}=1$. The vertices of $p(K)$ are the $V_{i}$ and the edges are the images, under $p f$, of $\left[t_{i-1}, t_{i}\right], 1 \leqq i \leqq 2 m$, where $t_{0}=0$. Observe that each vertex has degree four. If $R^{2}$ denotes the plane where $z=0$, then the complement, in $R^{2}$, of $p(K)$ is a disjoint union of $m+2$ domains (connected components) $X_{0}, X_{1}, \cdots, X_{m+1}$ where $X_{0}$ is the unbounded one. We call $p(K)$ common if

(i) the boundaries of any two distinct domains have at most one edge in common, and

(ii) each vertex is on the boundary of exactly four distinct domains.

Theorem A. Each polygonal, prime, nontrivial knot $K$ is equivalent to a polygonal knot $K_{0}$, in regular position, whose knot-diagram $p\left(K_{0}\right)$ is common. If, in addition, $p(K)$ is alternating, then $K_{0}$ can be chosen so that $p\left(K_{0}\right)$ is also alternating.

Proof. We may assume $K$ is in regular position $[2$, p. 7]. Use induction on the number of vertices of $p(K)$. If either (i) or (ii) fails to hold in $p(K)$, we shall see that $K$ can be replaced by an equivalent polygonal knot $K^{\prime}$, in regular position, such that $p\left(K^{\prime}\right)$ has fewer vertices than $p(K)$, and $p\left(K^{\prime}\right)$ is alternating if $p(K)$ is alternating. The proof will be completed by observing that a knot-diagram with at most one vertex corresponds to a trivial knot.

The failure of (i) is treated by an argument of Alexander [1, p. 279]. Now suppose (ii) fails at a vertex $V_{i}$. The domains which share $V_{i}$ as a boundary point can be listed in clockwise order as $X_{a}, X_{b}, X_{a}$, $X_{c}$ where $a, b, c$ are distinct. $V_{i}$ is the projection of an overcrossing $f\left(t_{j}\right)$ and undercrossing $f\left(t_{k}\right)$ for some $j, k$. Assume $t_{k}=1$ so that $0<t_{j}<1$. Then there is a simple closed curve $C$, in $R^{2}$, through $V_{i}$ such that $C-V_{i}$ lies in $X_{a}$ and the images, under $p f$, of $\left(0, t_{j}\right)$ and $\left(t_{j}, 1\right)$ lie in different components of $R^{2}-C$.

Let $K_{1}, K_{2}$ be the images, under $f$, of $\left[0, t_{j}\right]$ and $\left[t_{j}, 1\right]$, respectively. $K_{1}$ and $K_{2}$, minus their endpoints, lie on different sides of the cylinder $p^{-1}(C)$. Therefore $K$ is the result of joining together in succession the noninterlinking polygonal curves $K_{1}$ and $K_{2}$.

Since $K$ is nontrivial and prime, one of the curves, say $K_{1}$, is knotted and the other one is unknotted. Then $K$ is equivalent to the knot $K^{\prime \prime}=K_{1} \cup L_{1}$ where $L_{1}$ is the line segment from $f(1)$ to $f\left(t_{j}\right)$. Observe that $p\left(K^{\prime \prime}\right)=p\left(K_{1}\right)$. Finally $K^{\prime \prime}$ can be replaced by an equivalent polygonal knot $K^{\prime}$, in regular position, such that $p\left(K^{\prime}\right)$ has fewer vertices than $p(K)$. 
3. The Dehn presentation of $G$. Let $K$ be a polygonal knot in regular position. We use the notation from $\S 2$, so $p(K)$ has $m+2$ domains. Let $F$ be a free group on $m+2$ generators $x_{0}, x_{1}, \cdots, x_{m+1}$. (The generator $x_{j}$ corresponds to a path, starting at a distant base point on the $z$-axis, crossing the domain $X_{i}$, passing below the plane where $z=0$, and returning to the base point by way of $X_{0}$.) Each vertex $V_{i}$ of $p(K)$ determines one defining relator $r_{i}$ as follows.

The domains which share $V_{i}$ as a boundary point can be listed, possibly redundantly, in clockwise order $X_{a}, X_{b}, X_{c}, X_{d}$ where $a, b$, $c, d$ are not necessarily distinct. Observe that they are distinct when $p(K)$ is common. $V_{i}$ is the projection of some overcrossing $f\left(t_{j}\right)$ and some undercrossing $f\left(t_{k}\right)$. In $K$, we choose two arcs, an overpass $O$ containing $f\left(t_{j}\right)$ and an underpass $U$ containing $f\left(t_{k}\right)$. Let $K$ be given an orientation, with $O, U$ oriented accordingly. The subscripts in the above listing are to be chosen so that $X_{\dot{a}}$ meets $X_{\dot{b}}$ along an initial part of $O$. Put $r_{i}=x_{a} x_{b}^{-1} x_{c} x_{d}^{-1}$, which is a cyclically reduced word. Then the group of the $\operatorname{knot} K$ is

$$
G=\left\langle x_{0}, x_{1}, \cdots, x_{m+1} ; r_{1}, \cdots, r_{m}, x_{0}\right\rangle .
$$

The result of removing all traces of $x_{0}$ from this presentation can be described using the endomorphism $E$ of $F$ determined by $E\left(x_{0}\right)=1$ and $E\left(x_{j}\right)=x_{j}, 1 \leqq j \leqq m+1$.

REMARK. $G \cong\left\langle x_{1}, \cdots, x_{m+1} ; E\left(r_{1}\right), \cdots, E\left(r_{m}\right)\right\rangle$.

The promised group $H$ is given by

$$
H=\left\langle x_{0}, x_{1}, \cdots, x_{m+1} ; r_{1}, \cdots, r_{m}\right\rangle .
$$

LEMMA. $H$ is the free product of $G$ and the free group on one generator $x_{0}$.

Proof. Let $A$ be the automorphism of $F$ determined by $A\left(x_{0}\right)=x_{0}$ and $A\left(x_{j}\right)=x_{j} x_{0}, 1 \leqq j \leqq m+1$. With $E$ as before, some checking shows that the cyclically reduced form of $A\left(r_{i}\right)$ equals $E\left(r_{i}\right), 1 \leqq i \leqq m$. Thus

$$
H \cong\left\langle x_{0}, x_{1}, \cdots, x_{m+1} ; A\left(r_{1}\right), \cdots, A\left(r_{m}\right)\right\rangle
$$

and, by the remark,

$$
G \cong\left\langle x_{1}, \cdots, x_{m+1} ; A\left(r_{1}\right), \cdots, A\left(r_{m}\right)\right\rangle
$$

where the cyclically reduced form of each $A\left(r_{i}\right)$ does not involve $x_{0}$. This proves the lemma.

When $p(K)$ is common, the presentation of $H$ has the property $\mathrm{C}(4)$ which is defined and verified in Theorem $\mathrm{B}$. The definition of 
C(4) requires Lyndon's concept of a piece (relative to a subset $R$ of $F$ ) which is an element $u$ of $F$ such that $R$ contains two distinct elements with reduced forms $r=u v$ and $s=u w$.

TheOREM B. Let $K$ be a polygonal, prime knot, in regular position, with a common knot-diagram $p(K)$ which is alternating. Let $r_{1}, \cdots, r_{m}$ be the defining relators, of length 4 , in a Dehn presentation of the knot group of $K$. Let $R$ be the set consisting of $r_{i}, r_{i}^{-1}, 1 \leqq i \leqq m$, and all cyclically reduced conjugates of these elements. Then $R$ satisfies:

$\mathrm{C}(4)$ : No member of $R$ is a product of fewer than 4 pieces, and

$\mathrm{T}_{3}$ : if $r, s, t$ are in $R$, then in at least one of the products $r s$, st, tr there is no cancellation.

Proof. If $\mathrm{C}(4)$ fails, then there is some piece $x_{j} x_{k}^{-1}$ (or $x_{j}^{-1} x_{k}$ ) coming from two distinct elements $r, s$ in $R$. The boundaries of the corresponding domains $X_{j}, X_{k}$ have exactly one edge in common, since $p(K)$ is common. This edge is the image, under $p f$, of $\left[t_{i-1}, t_{i}\right]$, for some $i$, using the notation from $\$ 2$. Then $r, s$ correspond to vertices $p f\left(t_{i-1}\right)$ and $p f\left(t_{i}\right)$ where $f\left(t_{i-1}\right)$ and $f\left(t_{i}\right)$ are two successive overcrossings (or undercrossings). This is impossible since $p(K)$ is alternating. Thus $\mathrm{C}(4)$ holds.

If $\mathrm{T}_{3}$ fails, there exist domains $X_{i}, X_{j}, X_{k}$ such that $X_{i}, X_{j}$ have a common boundary edge $E_{i j}$ and the pairs $X_{j}, X_{k}$ and $X_{k}, X_{i}$ have the same property, for edges $E_{j k}, E_{k i}$, respectively. Let $P$ denote the planar graph determined by the vertices and edges of $p(K)$. Consider the graph $P^{*}$ which is dual to $P$. The faces $X_{i}, X_{j}, X_{k}$ and the edges $E_{i j}, E_{j k}, E_{k i}$ of $P$ correspond to parts of a triangle in $P^{*}$, namely, to vertices $X_{i}^{*}, X_{j}^{*}, X_{k}^{*}$ and edges $E_{i j}^{*}, E_{j k}^{*}, E_{k i}^{*}$, respectively. In $P^{*}$, there is a subgraph $S^{*}$ consisting of this triangle and all vertices and edges interior to it. Observe that each face of $S^{*}$ has four boundary edges since each vertex of $P$ has degree four. If $e^{*}, f^{*}$ denote the numbers of edges and faces, respectively, of $S^{*}$, we find $2 e^{*}=3+4 f^{*}$, an impossible equation. Thus, $\mathrm{T}_{3}$ holds, and we are done.

\section{Main result.}

Theorem C. Let $K$ be a tame, prime knot, in regular position, with a knot-diagram $p(K)$ which is alternating. Let $G$ be the knot group of $K$. Then the word and conjugacy problems for $G$ are solvable with respect to a Dehn presentation of $G$.

Proof. We may assume that $K$ is polygonal, nontrivial, and, by Theorem A, that $p(K)$ is common. By Theorem B, Lyndon's [4] Theorem II, and Schupp's [5] Theorem 4.1, the word and conjugacy 
problems are solvable for $H$. Hence, by the lemma, the same is true for $G$.

\section{REFERENCES}

1. J. W. Alexander, Topological invariants of knots and links, Trans. Amer. Math. Soc. 30 (1928), 275-306.

2. R. H. Crowell and R. H. Fox, Introduction to knot theory, Ginn, Boston, Mass., 1963. MR $26 \# 4348$.

3. M. Dehn, Über die Topologie des drei-dimensionalen Raumes, Math. Ann. 69 (1910), 137-168.

4. R. C. Lyndon, On Dehn's algorithm, Math. Ann. 166 (1966), 208-228. MR 35 \#5499.

5. P. E. Schupp, On Dehn's algorithm and the conjugacy problem, Math. Ann. 178 (1968), 119-130. MR 38 \#5901.

6. F. Waldhausen, The word problem in fundamental groups of sufficiently large irreducible 3-manifolds, Ann. of Math. (2) 88 (1968), 272-280. MR 30 \#2167.

Hawain loa College, Honolulu, Hawail 96810 